\title{
ON OPTIMIZED INEQUALITIES IN CONNECTION WITH COEFFICIENT BODIES OF BOUNDED UNIVALENT FUNCTIONS
}

\author{
OLLI TAMMI
}

\section{The first coefficient body}

1. Introduction. We shall consider the class $S(b)$ of functions $f$ which are bounded and univalent in the unit disc $U$ and satisfy the normalization conditions $|f(z)|<1, f(0)=0, f^{\prime}(0)>0$. Then

$$
f(z)=b z+b_{2} z^{2}+\ldots,
$$

where $0<b \leqq 1$. We set $b_{n} / b=a_{n}, n=2, \ldots$, and $a_{1}=1$.

The first coefficient body $\left(a_{2}, a_{3}\right)$ for $S(b)$-functions is characterized with the aid of the variational method in [1]. In [3] the resulting differential equation is used in showing that the question of finding boundary functions $f$ leads to the problem:

$$
\max \operatorname{Re}\left(\delta+2 x_{0} a_{2}\right), \quad \delta=a_{3}-a_{2}^{2},
$$

where $x_{0}$ is a complex parameter.

In [2] this question is studied in that subclass of $S(b)$ which consists of Löwnerfunctions. Thus, the inequalities obtained are directly applicable to the entire class $S(b)$, but the uniqueness of extremum functions is left open by this method. If the resulting inequalities are optimized with respect to the free parameters they characterize the coefficient body $\left(a_{2}, a_{3}\right)$ completely.

In the present paper we shall continue operating with Löwner-functions. We want to compare the optimized inequality mentioned above with an inequality obtained in an entirely different manner, namely by maximizing a proper functional under the side condition $a_{2}=$ constant. It appears that both methods lead to the same result. This remains to hold also for the next coefficient body $\left(a_{2}, a_{3}, a_{4}\right)$. Thus, the parallelism found seems to be independent of the index. Checking this by aid of direct calculations would be laborious. 
2. The sharp inequality for $\operatorname{Re}\left(\delta+2 x_{0} a_{2}\right)$.

Let

$$
u \mapsto \varkappa(u)=e^{i \vartheta(u)}, \quad u \in[b, 1],
$$

be the generating function of Löwner-functions; $x$ is supposed to be piecewise continuous. For the first coefficients we have

$$
a_{2}=-2 \int_{b}^{1} x d u, \quad \delta=a_{3}-a_{2}^{2}=-2 \int_{b}^{1} u x^{2} d u .
$$

We consider the combination

$$
\delta+2 x_{0} a_{2}=-2 \int_{b}^{1}\left(u x^{2}+2 x_{0} x\right) d u
$$

and vary this by replacing $\vartheta$ by $\tilde{\vartheta}$ so that

$$
\tilde{\vartheta}(u)=\vartheta(u)+\varepsilon \eta(u) \text {. }
$$

Here $\eta$ is of the same type as $\vartheta$ and $\varepsilon$ is a small real number. Because

$$
\tilde{\varkappa}=\varkappa+i \varepsilon x \eta+O\left(\varepsilon^{2}\right)
$$

we have for (3)

$$
\tilde{\delta}+2 x_{0} \tilde{a}_{2}=\delta+2 x_{0} a_{2}-4 \varepsilon i \int_{b}^{1}\left(u \varkappa^{2}+x_{0} \varkappa\right) \eta d u+O\left(\varepsilon^{2}\right) .
$$

The freedom of $\eta$ allows to extract from this the following necessary condition for $\varkappa$ to be extremal with respect to the problem (2):

$$
\operatorname{Im}\left(u x^{2}+x_{0} x\right)=0
$$

Let us write this in two equivalent forms :

$$
\begin{gathered}
u\left(\varkappa^{2}-\varkappa^{-2}\right)+x_{0} \varkappa-\bar{x}_{0} \varkappa^{-1}=0, \\
u \sin 2 \vartheta-C_{1} \sin \vartheta-C_{2} \cos \vartheta=0, \quad C=-x_{0}=C_{1}+i C_{2} .
\end{gathered}
$$

Observe that integration of $(5)$ over $[b, 1]$ shows that in the extremum case

$$
\operatorname{Im}\left(\delta+x_{0} a_{2}\right)=0 .
$$

From this onwards we shall keep $a_{2}$ constant in the inequality found:

(9) $\operatorname{Re} \delta \leqq 2 C_{1} \operatorname{Re} a_{2}-2 C_{2} \operatorname{Im} a_{2}+4 \int_{b}^{1}\left(-\frac{u}{2} \cos 2 \vartheta_{0}+C_{1} \cos \vartheta_{0}-C_{2} \sin \vartheta_{0}\right) d u$

$$
=M\left(C_{1}, C_{2}\right)=M \text {. }
$$

Here $\vartheta_{0}$ is the extremum function determined by (7):

$$
\vartheta_{0}=\vartheta_{0}\left(u, C_{1}, C_{2}\right) \text {. }
$$


3. Optimization with respect to $x_{0}$. We now optimize the inequality (9) with respect to $x_{0}$ or with respect to the two parameters $C_{1}$ and $C_{2}$ by requiring that

$$
\begin{aligned}
& \frac{\partial M}{\partial C_{1}}=2 \operatorname{Re} a_{2}+4 \int_{b}^{1}\left\{\left[u \sin 2 \vartheta_{0}-C_{1} \sin \vartheta_{0}-C_{2} \cos \vartheta_{0}\right] \frac{\partial \vartheta_{0}}{\partial C_{1}}+\cos \vartheta_{0}\right\} d u=0, \\
& \frac{\partial M}{\partial C_{2}}=-2 \operatorname{Im} a_{2}+4 \int_{b}^{1}\left\{\left[u \sin 2 \vartheta_{0}-C_{1} \sin \vartheta_{0}-C_{2} \cos \vartheta_{0}\right] \frac{\partial \vartheta_{0}}{\partial C_{2}}-\sin \vartheta_{0}\right\} d u=0 .
\end{aligned}
$$

Hence, according to (7) the optimizing conditions for the extremal $\vartheta_{0}$ assume the form

$$
\operatorname{Re} a_{2}=-2 \int_{b}^{1} \cos \vartheta_{0} d u, \quad \operatorname{Im} a_{2}=-2 \int_{b}^{1} \sin \vartheta_{0} d u .
$$

The inequality (9), under these conditions, assumes the form

$$
\operatorname{Re} \delta \leqq-2 \int_{b}^{1} u \cos 2 \vartheta_{0} d u
$$

showing that equality is reached for the extremal $\vartheta_{0}$.

We may express the result in closed integrated form by aid of the parameters

$$
\omega=-\vartheta_{0}(b), \quad \alpha=-\vartheta_{0}(1) .
$$

Condition (7) determines the inverse connection in explicit form $\left(\vartheta_{0}=\vartheta\right)$ :

$$
u=u(\vartheta)=\frac{C_{1}}{2 \cos \vartheta}+\frac{C_{2}}{2 \sin \vartheta},
$$

which allows the following integrations

$$
\begin{aligned}
\operatorname{Re} a_{2} & =-2 \int_{b}^{1} \cos \vartheta d u=C_{2} \int_{-\omega}^{-\alpha} \cot ^{2} \vartheta d \vartheta-C_{1} \int_{-\omega}^{-\alpha} \tan \vartheta d \vartheta \\
& =C_{2}(\cot \alpha-\cot \omega+\alpha-\omega)+C_{1} \log \frac{\cos \alpha}{\cos \omega},
\end{aligned}
$$

$$
\begin{aligned}
\operatorname{Im} a_{2} & =-2 \int_{b}^{1} \sin \vartheta d u=C_{2} \int_{-\omega}^{-\alpha} \cot \vartheta d \vartheta-C_{1} \int_{-\omega}^{-\alpha} \tan ^{2} \vartheta d \vartheta \\
& =C_{2} \log \frac{\sin \alpha}{\sin \omega}+C_{1}(\tan \alpha-\tan \omega-\alpha+\omega) .
\end{aligned}
$$

Condition (7) implies two more connections

$$
b \sin 2 \omega-C_{1} \sin \omega+C_{2} \cos \omega=0, \quad \sin 2 \alpha-C_{1} \sin \alpha+C_{2} \cos \alpha=0 .
$$


These conditions determine $C_{1}$ and $C_{2}$ in $\alpha$ and $\omega$ which further are determined by $a_{2}$. The inequality (11) assumes in these quantities the form

$$
\begin{aligned}
\operatorname{Re} \delta & \leqq 1-b^{2}+C_{1}^{2} \log \frac{\cos \alpha}{\cos \omega}-C_{2}^{2} \log \frac{\sin \alpha}{\sin \omega}-\frac{C_{2}^{2}}{2}\left(\sin ^{-2} \alpha-\sin ^{-2} \omega\right) \\
& -C_{1} C_{2}(\cot \omega-\cot \alpha)-2 C_{1} C_{2}(\omega-\alpha) .
\end{aligned}
$$

Clearly, all the results can still be generalized by the rotation $\tau f\left(\tau^{-1} z\right),|\tau|=1$, i.e. instead of $a_{2}$ and $\delta$ we may put $\tau^{-1} a_{2}$ and $\tau^{-2} \delta$ in the above conditions. The parameter $\tau$ has a geometrical meaning in connection with the extremum domain [3].

4. Maximizing $\operatorname{Re} \delta$ with the side condition $a_{2}=c$. In Löwner's class we may specialize $x$ as a step-function

$$
\varkappa=\varkappa_{v}=e^{i \vartheta_{v}}, \quad x \in\left(x_{v-1}, x_{v}\right] ; \quad v=1, \ldots, N ; \quad x_{0}=b, \quad x_{N}=1 .
$$

This gives us

$$
\delta=-\sum_{1}^{N}\left(x_{v}^{2}-x_{v-1}^{2}\right) x_{v}^{2}, \quad a_{2}=-2 \sum_{1}^{N}\left(x_{v}-x_{v-1}\right) x_{v} .
$$

Consider the problem of maximizing $\operatorname{Re} \delta$ with the side condition $a_{2}=c=$ constant. This implies introducing the functional $F$ with a Lagrange multiplier $\lambda$ :

$$
F=\operatorname{Re}\left\{\delta+2 \lambda\left(a_{2}-c\right)\right\}=\operatorname{Re}\left\{-\sum_{1}^{N}\left(x_{v}^{2}-x_{v-1}^{2}\right) x_{v}^{2}+2 \lambda\left(-2 \sum_{1}^{N}\left(x_{v}-x_{v-1}\right) x_{v}-c\right)\right\},
$$

where

$$
c=-2 \sum_{1}^{N}\left(x_{v}-x_{v-1}\right) x_{v} .
$$

Thus the necessary extremum conditions for $x$ are

$$
\frac{\partial F}{\partial \vartheta_{\mu}}=-2 i\left[\left(x_{\mu}^{2}-x_{\mu-1}^{2}\right)\left(\chi_{\mu}^{2}-x_{\mu}^{-2}\right)+2\left(x_{\mu}-x_{\mu-1}\right)\left(\lambda x_{\mu}-\bar{\lambda} x_{\mu}^{-1}\right)\right]=0 .
$$

The limit process $N \rightarrow \infty$ hence gives to the necessary conditions the form

$$
u\left(x^{2}-x^{-2}\right)+\lambda x-\bar{\lambda} x^{-1}=0, \quad-2 \int_{b}^{1} x d u=c,
$$

i.e. we are led to the previous conditions (6) and (10).

As was seen, the problem

$$
\max \operatorname{Re} \delta, \quad a_{2}=\text { constant, }
$$

is solved by the same necessary conditions which were met with in connection with the sharp estimate $\operatorname{Re}\left(\delta+2 x_{0} a_{2}\right)$ having an optimized parameter $x_{0}$. We want to show that a similar situation remains to hold for the next coefficient body, too. 


\section{The second coefficient body}

1. The sharp optimized inequality. The Löwner-coefficients satisfy the following identity

$$
\delta_{2}+2 \lambda \delta_{1}+\mu a_{2}=-2 \int_{b}^{1}\left[A^{2}+\left(\mu-\lambda^{2}\right) \chi\right] d u .
$$

Here

$$
\begin{gathered}
\delta_{2}=a_{4}-2 a_{2} a_{3}+\frac{13}{12} a_{2}^{3}, \\
\delta_{1}=a_{3}-\frac{3}{4} a_{2}^{2}=2 \int_{b}^{1} x\left(\int_{u}^{1} x d v-u x\right) d u, \\
A=x^{1 / 2} \alpha, \quad \alpha=\int_{u}^{1} x d v-u x-\lambda .
\end{gathered}
$$

In the special case $\mu=\lambda^{2}$ this identity was introduced by 0 . Jokinen who pointed out that the second Power inequality is a direct consequence of it.

Let us keep $\lambda$ and $\mu$ free complex parameters and consider the problem

$$
\max \operatorname{Re}\left(\delta_{2}+2 \lambda \delta_{1}+\mu a_{2}\right)=\max \left[-2 \int_{b}^{1} \operatorname{Re}\left\{A^{2}+\left(\mu-\lambda^{2}\right) x\right\} d u\right] .
$$

Again, we derive the necessary extremum condition for $x$ by using step functions. Thus, we consider the functional

where

$$
G=\int_{b}^{1}\left[A^{2}+\left(\mu-\lambda^{2}\right) \varkappa\right] d u=\sum_{1}^{N}\left[A_{v}^{2}+\left(\mu-\lambda^{2}\right) \varkappa_{v}\right] \Delta_{v},
$$

Because

$$
\Delta_{v}=x_{v}-x_{v-1}, \quad A_{v}=k_{v}^{1 / 2} \alpha_{v} .
$$

we have

$$
\frac{\partial}{\partial \vartheta_{k}} \operatorname{Re} G=\operatorname{Re} \frac{\partial G}{\partial \vartheta_{k}}, \quad \frac{\partial}{\partial \vartheta_{k}}=i \varkappa_{k} \frac{\partial}{\partial \varkappa_{k}},
$$

$$
\frac{\partial}{\partial \vartheta_{k}} \sum_{1}^{N} A_{v}^{2} \Delta_{v}=i \Delta_{k}\left(2 \varkappa_{k} \sum_{\mu=1}^{k} \alpha_{\mu} \varkappa_{\mu} \Delta_{\mu}+\alpha_{k}^{2} \varkappa_{k}-2 x_{k} \alpha_{k} \varkappa_{k}^{2}\right) .
$$

Hence, the following necessary extremum conditions hold:

$$
\begin{gathered}
\frac{1}{\Delta_{k}} \operatorname{Re} \frac{\partial}{\partial \vartheta_{k}} \sum_{1}^{N}\left[A_{v}^{2}+\left(\mu-\lambda^{2}\right) \varkappa_{v}\right] \Delta_{v} \\
=-\operatorname{Im}\left\{2 \varkappa_{k} \sum_{\mu=1}^{k} \alpha_{\mu} \varkappa_{\mu} \Delta_{\mu}+\alpha_{k}^{2} \varkappa_{k}-2 x_{k} \alpha_{k} \varkappa_{k}^{2}+\left(\mu-\lambda^{2}\right) \varkappa_{k}\right\}=0 .
\end{gathered}
$$


The limit process gives the integral form for the necessary condition:

$$
\operatorname{Im}\left\{2 \varkappa \int_{b}^{u} \alpha x d v+\alpha^{2} x-2 u \alpha \varkappa^{2}+\left(\mu-\lambda^{2}\right) x\right\}=0,
$$

where

$$
\alpha=\int_{u}^{1} x d v-u x-\lambda
$$

Inserting the expression of $\alpha$, the result assumes the form

$$
\begin{gathered}
\operatorname{Im}\left\{x\left(\int_{b}^{1} x d u\right)^{2}-4 u x^{2} \int_{u}^{1} x d v-2 x \int_{b}^{u} u x^{2} d v\right. \\
\left.+3 u x^{3}-2 \lambda x \int_{b}^{1} x d u+4 \lambda u x^{2}+\mu x\right\}=0 .
\end{gathered}
$$

Integration of this over $[b, 1]$ yields in the extremum case

$$
\operatorname{Im}\left(3 \delta_{2}+4 \lambda \delta_{1}+\mu a_{2}\right)=0 .
$$

We may, of course, use also the variation (4) of $\chi$, which gives us a variational formula $\left(\beta=\int_{u}^{1} x \eta d v-u x \eta\right)$ :

$$
\begin{gathered}
\int_{b}^{1}\left[\tilde{A}^{2}+\left(\mu-\lambda^{2}\right) \tilde{x}\right] d u-\int_{b}^{1}\left[A^{2}+\left(\mu-\lambda^{2}\right) x\right] d u \\
=i \varepsilon \int_{b}^{1}\left[2 \alpha \beta \varkappa+\alpha^{2} x \eta+\left(\mu-\lambda^{2}\right) \varkappa \eta\right] d u+O\left(\varepsilon^{2}\right) \\
=i \varepsilon \int_{b}^{1}\left[2 x \int_{b}^{1} \alpha x d u+2 x \int_{1}^{u} \alpha x d v-2 \alpha u x^{2}+\alpha^{2} x+\left(\mu-\lambda^{2}\right) x\right] \eta d u+O\left(\varepsilon^{2}\right) .
\end{gathered}
$$

Hence, the factor of $\eta$ gives again the necessary condition (21) for that $\varkappa$ which is an extremal for the problem (19).

Observe that in the conditions (18) we differentiate with respect to $\vartheta_{k}$ in the sum. In the integral form this means differentiation with respect to $\vartheta$ for a fixed $u$. Thus, we may write the necessary condition (21) in the equivalent form

$$
\frac{\partial}{\partial \vartheta} \int_{b}^{1} \operatorname{Re}\left\{A^{2}+\left(\mu-\lambda^{2}\right) x\right\} d u=0 .
$$

The problem (19) introduces the following sharp inequality:

$$
\begin{aligned}
\operatorname{Re} \delta_{2} & \leqq-2 \operatorname{Re}\left(\lambda \delta_{1}\right)-\operatorname{Re}\left(\mu a_{2}\right)-2 \int_{b}^{1} \operatorname{Re}\left\{A^{2}+\left(\mu-\lambda^{2}\right)\right\}_{0} d u \\
& =M\left(\lambda_{1}, \lambda_{2}, \mu_{1}, \mu_{2}\right)=M ;
\end{aligned}
$$


where

$$
\lambda=\lambda_{1}+i \lambda_{2}, \quad \mu=\mu_{1}+i \mu_{2} .
$$

Here \{\}$_{0}$ is determined by $\varkappa=\chi_{0}$ obtained as solution of (19) or of the equivalent form (24). In $M$ we consider $\delta_{1}$ and $a_{2}$ to be given fixed numbers and $\vartheta_{0}$, determined by (24), to be a function

$$
\vartheta_{0}=\vartheta_{0}\left(u, \lambda_{1}, \lambda_{2}, \mu_{1}, \mu_{2}\right) .
$$

The inequality (25) is now optimized with respect to the parameters. First we determine by using (24)

$$
\begin{aligned}
\frac{\partial M}{\partial \lambda_{1}}= & \frac{\partial M}{\partial \lambda}=-\delta_{1}-\bar{\delta}_{1}-2 \frac{\partial}{\partial \vartheta_{0}} \int_{b}^{1} \operatorname{Re}\left\{A^{2}+\left(\mu-\lambda^{2}\right) x\right\}_{0} d u \frac{\partial \vartheta_{0}}{\partial \lambda_{1}} \\
& \left.-\int_{b}^{1}\left[\frac{\partial}{\partial \lambda} A^{2}-2 \lambda x+\overline{\left(\frac{\partial}{\partial \lambda} A^{2}-2 \lambda x\right.}\right)\right] d u \\
= & \left.-\delta_{1}-\bar{\delta}_{1}-\int_{b}^{1}\left[\frac{\partial}{\partial \lambda} A^{2}-2 \lambda x+\overline{\left(\frac{\partial}{\partial \lambda} A^{2}-2 \lambda x\right.}\right)\right] d u .
\end{aligned}
$$

Here

$$
\frac{\partial}{\partial \lambda} A^{2}-2 \lambda x=2 A\left(-x^{1 / 2}\right)-2 \lambda \varkappa=-2 \varkappa\left(\int_{b}^{1} x d v-u x\right)
$$

and thus, according to (18),

$$
\frac{\partial M}{\partial \lambda_{1}}=-2 \operatorname{Re} \delta_{1}+2 \operatorname{Re} \delta_{1}^{0},
$$

where $\delta_{1}^{0}$ is determined by $\varkappa_{0}$. Hence, the optimizing condition $\partial M / \partial \lambda_{1}=0$ implies that

Similarly

$$
\operatorname{Re} \delta_{1}=\operatorname{Re} \delta_{1}^{0} .
$$

$$
\begin{aligned}
\frac{\partial M}{\partial \mu_{1}}=\frac{\partial M}{\partial \mu}= & -\frac{1}{2}\left(a_{2}-\bar{a}_{2}\right)-2 \frac{\partial}{\partial \vartheta_{0}} \int_{b}^{1} \operatorname{Re}\left\{A^{2}+\left(\mu-\lambda^{2}\right) \chi\right\}_{0} d u \frac{\partial \vartheta_{0}}{\partial \mu_{1}} \\
& -\int_{b}^{1}(\varkappa+\bar{\chi}) d u=-\operatorname{Re} a_{2}+\operatorname{Re} a_{2}^{0},
\end{aligned}
$$

i.e. the condition $\partial M / \partial \mu_{1}=0$ implies that

$$
\operatorname{Re} a_{2}=\operatorname{Re} a_{2}^{0} .
$$

Corresponding calculations show that the conditions $\partial M / \partial \lambda_{2}=0$ and $\partial M / \partial \mu_{2}=0$ yield

$$
\operatorname{Im} \delta_{1}=\operatorname{Im} \delta_{1}^{0}
$$

$$
\operatorname{Im} a_{2}=\operatorname{Im} a_{2}^{0} .
$$


Altogether, we are led to the optimizing conditions (26)-(29) which are similar to those in (10).

2. Maximizing $\operatorname{Re} \delta_{2}$ with the side conditions $\delta_{1}=c_{1}, a_{2}=c_{2}$. The above results clearly suggest repeating the procedure of Section I.4. We have to find a necessary condition for $\varkappa$ maximizing $\operatorname{Re} \delta_{2}$ with the side conditions $\delta_{1}=c_{1}=$ constant and $a_{2}=c_{2}=$ constant. Again, let us introduce the functional

$$
\begin{aligned}
H & =\operatorname{Re}\left\{\delta_{2}+2 \lambda\left(\delta_{1}-c_{1}\right)+\mu\left(a_{2}-c_{2}\right)\right\} \\
& =\operatorname{Re}\left\{\delta_{2}+2 \lambda \delta_{1}+\mu a_{2}\right\}-\operatorname{Re}\left(2 \lambda c_{1}+\mu c_{2}\right) .
\end{aligned}
$$

Because the second part of this expression is a constant, we obtain the same necessary extremum condition (21) as in the previous problem of Section I.4. The side conditions are actually those of (26)-(29).

Hence we have checked that also in the present case the proper optimized inequality and the corresponding extremum problem with side conditions do agree.

In Section I.3 the equality case, corresponding to the boundary function of the coefficient body, was parametrized by using integrated equations and two parameters $\alpha$ and $\omega$. In the present case integration in closed form is no more possible. However, also here the problem can be considered to be parametrized in $\alpha$ and $\mu$. The generating function $x$ belonging to these parameters is determined by (22) and the side conditions (26)-(29). The corresponding optimized inequality

$$
\operatorname{Re} \delta_{2} \leqq \operatorname{Re} \delta_{2}^{0}
$$

gives the range of $\delta_{2}$. Observe finally that as before, we can add a normalizing parameter $\tau$ by aid of the rotation $\tau f\left(\tau^{-1} z\right)$.

\section{References}

[1] CharzyŃski, Z., and W. Janowski: Domaine de variation des coefficients $A_{2}$ et $A_{3}$ des fonctions univalentes et bornées. - Bull. Soc. Sci. Lettres Lódź, 10 no. 4, 1959, 1-29.

[2] HAARIO, H., and O. JoKINEN: On the use of Löwner's functions for finding inequalities for the first coefficient body of bounded univalent functions. - Reports of the Dept. of Mathematics, University of Helsinki, Ser. A No. 14, 1977, 1-13.

[3] Tаммі, O.: Extremum problems for bounded univalent functions. - Lecture Notes in Mathematics 646, Springer-Verlag, Berlin-Heidelberg-New York, 1978.

University of Helsinki

Department of Mathematics

SF-00100 Helsinki 10

Finland

Received 17 March 1978 